\title{
The Watershed Transformation for Multiresolution Image Segmentation
}

\author{
S. Wegner , T. Harms, J. H. Builtjes, H. Oswald, E. Fleck \\ Deutsches Herzzentrum Berlin, Augustenburger Platz 1, \\ D-13353 Berlin, Germany
}

\begin{abstract}
For a hyperthermia planning environment the main requirements for the following numerical computation and visualization of the temperature field are the separation of several anatomical objects. Since different contrast objects cannot be seen at the same resolution, a segmentation technique using multiresolution segmentation planes is described here. The computation of these planes is based on the technique of region growing using watersheds in digital images. First, the watershed transformation is applied on the gradient image. The resulting image is then transformed into a graph on which a region growing process is performed. The iteration of these two actions results in hierarchical segmentation planes which differ in region amount and region size. Consequently low contrast objects can be separated in the lower planes whereas high contrast objects can be extracted in the higher planes.
\end{abstract}

\section{Introduction}

The main aspect of hyperthermia planning in cancer therapy is the patient-specific computation of the physical relevant temperature field parameters. Moreover, these computations are the basis for the optimization of the temperature treatment determining the optimal values for the variable technical parameters. This planning requires the following steps: acquisition of the Computer Tomography images, segmentation of these images, computation and visualization of the temperature field and finally the optimization of this field. Obviously, one of the main requirements for the succeeding steps is the separation of several anatomical objects, for instance tumor, skin, bones, different organs, fat, muscle. In particular the extraction of low contrast or even diffuse objects is required. Since these objects are best seen at different resolutions, multiresolution segmentation planes are computed. In the lower planes low contrast objects can be separated, whereas in the higher planes higher contrast objects can be extracted.

The construction of the multiresolution segmentation planes is based on the concept of region growing using watersheds in digital images. A preprocessing segmentation is obtained using the watershed transformation on the gradient image. The resulting image is then transformed into a graph on which the region growing is performed. This can be seen as the watershed transformation on graphs. The gradient of the graph is computed and regions with similar graylevels are merged to one region. The iteration of this procedure leads to the multiresolution segmentation planes. 
These planes form the basis of an interactive segmentation tool for the hyperthermia planning environment. The user can select the high and low contrast objects in different planes for a final image segmentation. If an object is separated into several regions the user has the possibility of merging these regions. For most images a sufficient segmentation result is achieved when applying these two actions. However, for some diffuse objects interactively obtained contours are necessary.

\section{Watershed Transformation}

Watersheds were introduced in the field of mathematical morphology where grayscale pictures are often considered as topographic reliefs. In the topographic representation of an image, the numerical value of each pixel represents the elevation at this point. A drop of water falling on the topographic relief flows down until it reaches a regional minimum. A region or catchment basin associated with a minimum is given by the amount of points from which a drop of water flows down until this minimum. A watershed is defined as a side from where a drop of water can flow down to two minimas. The optimal contours of the image are these watersheds.

Employing watersheds to image segmentation the gradient of the image has to be interpreted as a relief. The original graylevel image is inappropriate because the amount of the graylevel elevation does not allow the unmistakable classification of valleys to regions and elevations to edges. The gradient of an image provides the absolute amount of inhomogeneities in an image. Homogeneous regions of the image are characterized by low contrast and hence a low gradient, whereas the high values of the gradient image are created by the high contrast contours of the original image.

The algorithmic computation of the watershed approach can be interpreted as an immersion process [Beu 90] [Vin 91]. Drilling holes in each regional minimum of the relief, the surface is immersed into a lake with uniform vertical speed. It is supposed that the immersion speed is slow enough to ensure a constant water level in all the basins. Starting from the minima of the lowest altitude, the water will fill up the different catchment basins. To avoid the confluence of the flood, dams are built along the lines where the water of two different catchment basins would merge. At the end of this immersion procedure each minimum is completely surrounded by dams, which delimit its associated catchment basins. These dams correspond to the watersheds of the image and hence the contours of the image.

\section{Multiresolution Image Planes}

The described technique results in regions with good located closed contours, but it leads to a strong oversegmentation, i.e., the interesting contours can not be seen clearly because of many irrelevant contours. A solution is the application of the watershed transformation on graphs. Instead of using the numerical value of each pixel for the topographic representation here presegmented regions are considered. This region merging can be interpreted as a region growing process on graphs where the regions are represented as nodes and the relations between them as arcs:

Let $G=(V, E)$ be a decimal non-oriented, non-reflexive graph, where $V$ is the set of nodes and $E$ is the set of arcs.

For a region graph the nodes describe regions and the arcs represent the neighborhood relationship between these regions (Figure 1). 


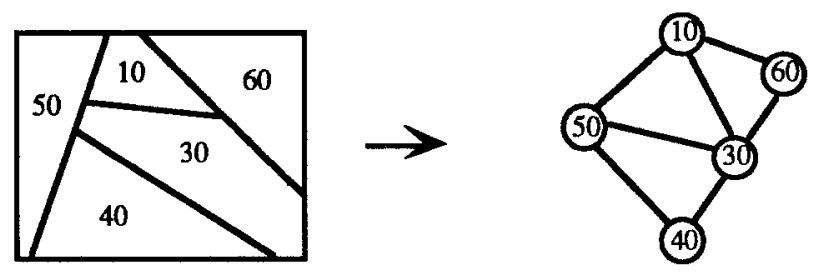

Fig. 1. Mosaic image and region graph

The regions are obtained using the watershed transformation on the gradient image. Each region is then characterized by only one graylevel achieving a so called mosaic image. The selected graylevels should be similar to the graylevels of the original image. Meyer and Beucher [Beu 90] chose the graylevel of the accompanying gradient minimum since this should represent the homogenest part of the region. We recognized that this is not always the best characterization of a region according to the segmentation result. Hence, in our environment the user has the possibility to select an appropriate criterion: homogenest part, minimum, maximum, average or median of the region.
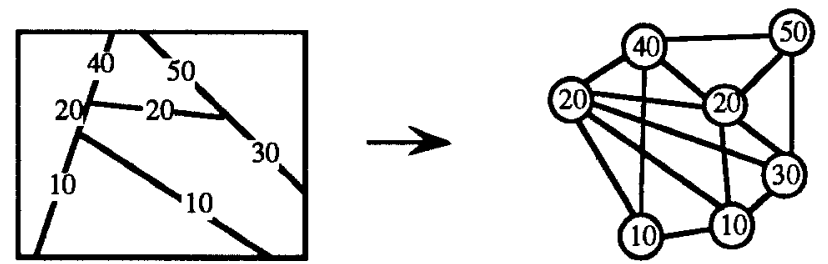

Fig. 2. Mosaic image - Figure 1 - with edge values and contour graph

Representing the regions as nodes in a graph leads to the problem that the watershed transformation cannot be performed directly on the graph. The reason is that this graph includes no information about the graylevel differences of neighbor regions. A solution could be an approximation, for instance the average, of all graylevel neighbor differences [Zeh 93]. However, since this is only an approximation of the graylevel differences, the finally obtained contours are often inexact. Zehetbauer [Zeh 93] suggested a so called contour graph as a model for the gradient. Every arc in the region graph - edge in the mosaic image - is represented as a node in the contour graph. The value of the node is the absolute difference of the two neighbor regions. Then all nodes belonging to one region are linked by arcs (Figure 2).

Considering this model some additional difficulties appear because in this representation a node is constructed of two regions. For instance, if the upper and lower region contours have been labeled as watersheds and the left and right as $\mathrm{x}$ or $y$, then the region could be labeled $x$ or $y$ (Figure 3). A solution is obtained by a combination of the region graph and the contour graph. On the basis of the labeled contour graph the nodes of the region graph are labeled with the most often contour label. If there are equally occurring labels the lowest label is selected.

The multiresolution segmentation planes are obtained by computing iteratively the mosaic image and applying the watershed transformation on the contour graph of this mosaic image. This process is continued until only one region remains. This action results in mosaic images of order $\mathrm{n}$ representing the $\mathrm{n}$-th segmentation plane. 
These planes are the basis of an interactive segmentation tool for the hyperthermia planning environment. The user has the possibility to select objects in different planes for a final image segmentation. The advantage here is that the different contrast objects that have been separated in different planes are unified in one image. Moreover, if an object is separated into several regions the user has the possibility of merging these regions for the final image. This is again possible in different planes. For most images a sufficient segmentation result is achieved when applying these two actions. However, diffuse objects are nearly impossible to separate even at different resolution planes. Hence, the user has additionally the possibility to obtain contours interactively.

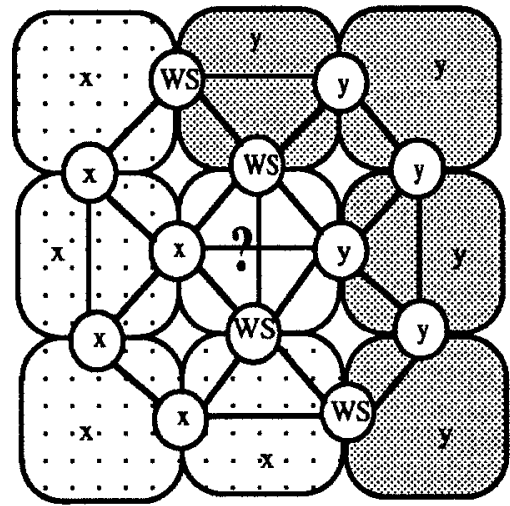

Fig. 3. Mosaic image and contour graph

\section{Results and Conclusion}

The upper image of Figure 4 shows a typical CT image slice acquired for a patientspecific hyperthermia planning, whereas the lower image shows the segmentation result of this slice. The different anatomical object are merged together from different resolution planes without interactively obtained contours. Only those objects are selected that are relevant for the following numerical computation of the temperature field parameters. The segmentation result is sufficient for most anatomical objects of the image but some contour corrections have to be made for the very low contrast objects. Despite these small corrections a user of this segmentation environment has the advantage to obtain most of the relevant contours automatically.

In order to prove the generality of this segmentation approach Figure 5 shows an example from a different application domain. The upper image presents the original $\mathrm{X}$-ray ventriculogram whereas the lower image shows the obtained segmentation result. Current efforts focus on the model based selection of the high and low contrast objects in different planes. Furthermore, the approach should be extended to a 3D segmentation tool.

\section{References}

[Beu 90] S. Beucher, F. Meyer: Morphological Segmentation, Journal of Visual Communication and Image Representation, Vol. 1, No. 1, pp 21-46, Sept. 1990 


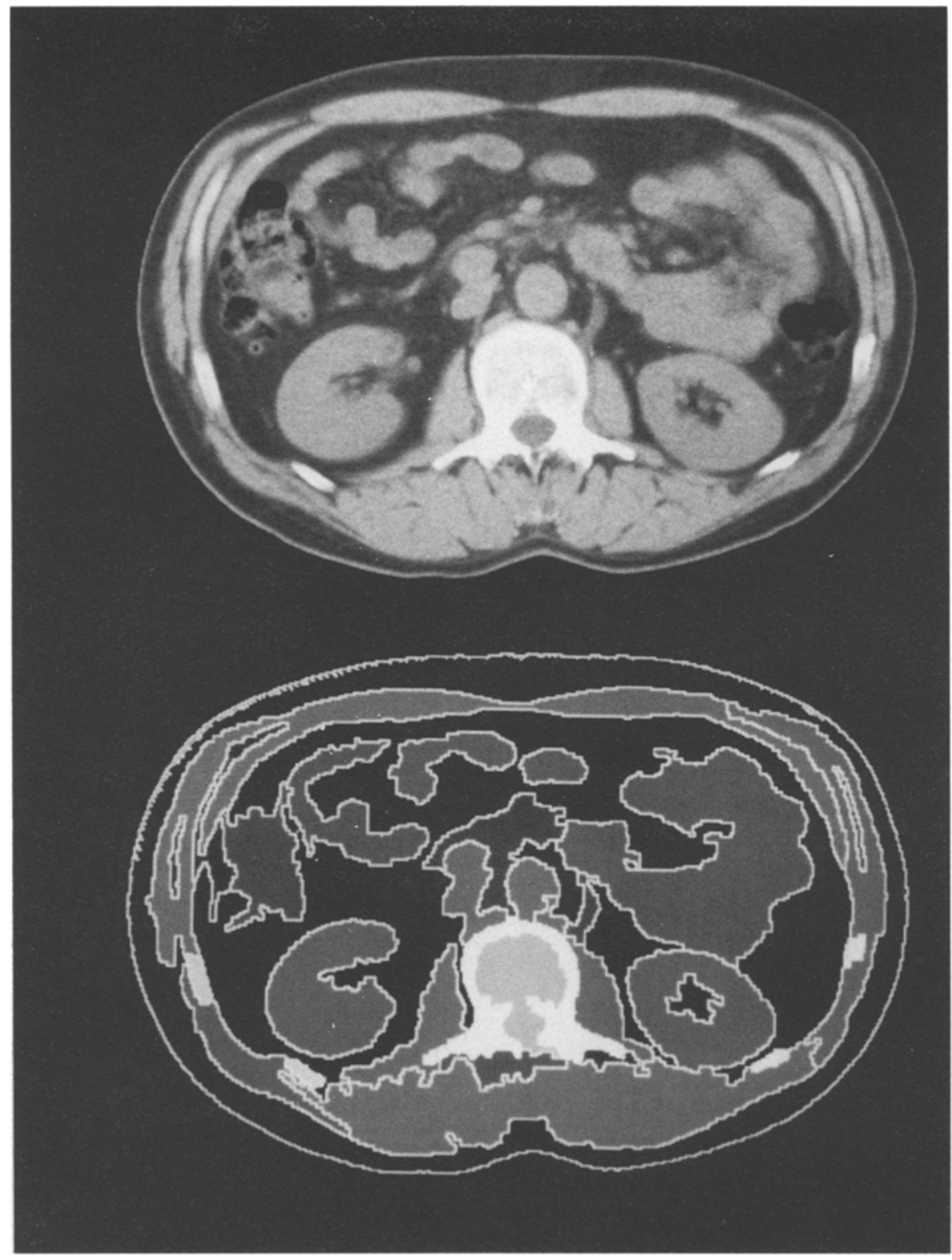

Fig 4. CT image slice and segmentation result

[Vin 89] L. Vincent: Graphs and Mathematical Morphology, Signal Processing 16, pp 365-388, 1989

[Vin 91] L. Vincent, P. Soille: Watersheds in Digital Spaces: An Efficent Algorithm Based on Immersion Simulation, IEEE Transactions on Pattern Analysis and Machine Intelligence, Vol.13, No.6, pp 583-598, Jun. 1991 
[Zeh 93] S. Zehetbauer, U. Meyer-Gruhl: Segmentierung und Analyse drei- und vierdimensionaler Ultraschalldatensätze, S. J. Pöppl, H. Handels, Mustererkennung 1993, pp 118-125

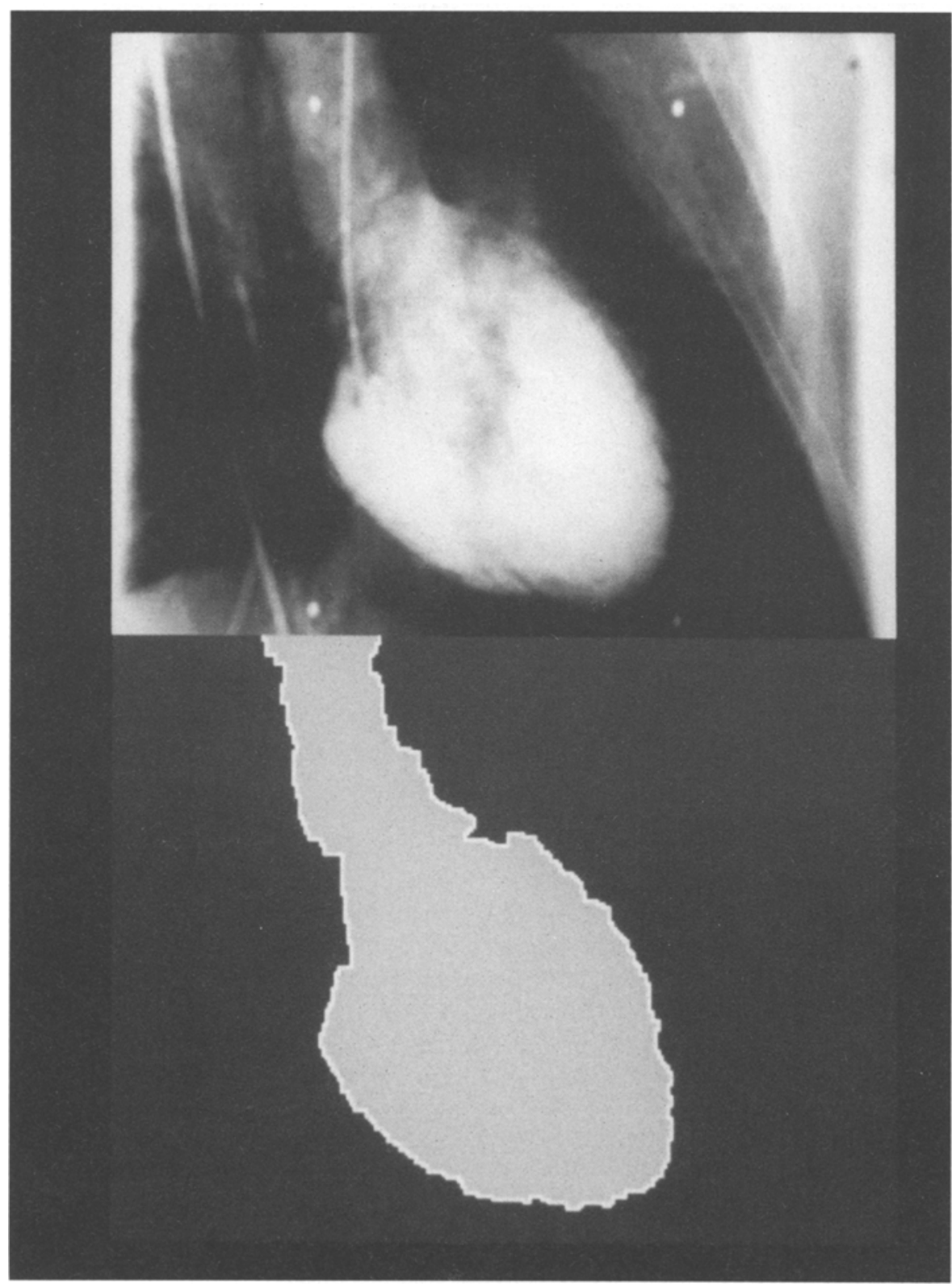

Fig. 5. X-ray ventriculogram and segmentation result 